Books, videos, cd-roms, dvds and any other relevant items submitted for a review in the BDJ should be addressed to:

Mike Grace, Editor, British Dental Journal, 64 Wimpole Street WIG 8YS

\section{Complete Dentures: From Planning to Problem Solving}

\author{
P. F. Allen and S. McCarthy \\ Surrey:, Quintessence, 2003 \\ price $€ 28$, pp 119 \\ ISBN 1850970645
}

It is well recognised that the need for complete dentures is declining due to improvements in dental health. As a result, a large proportion of the dental profession now has less experience in providing complete dentures than in the past. However, the 1998 Adult Dental Health Survey estimates that at present there are four to five million edentulous adults in England and Wales: so there is still significant demand.

In Complete Dentures: From Planning to Problem Solving it is pleasing to see the opening chapter focus on how to manage the transition to edentulousness. The authors explain that this is a critical period for the older adult with a failing dentition; if handled well, this phase can contribute considerably to a successful long-term outcome. If handled badly, the patient may never adapt successfully to wearing complete dentures.

Each of the 10 chapters commence with a brief statement of the aims and anticipated outcomes, and end with key clinical points and conclusions. The bulk of the book deals with the planning, construction and review of complete dentures, with the addition of a chapter on the role of the replica or copy denture technique in the management of the edentulous patient. A final chapter discusses the place of osseointegrated prostheses in the treatment of edentulism.

At just over 100 pages, the text is easy to read with an emphasis on sensible, practical solutions to the basic challenges of constructing complete dentures. This is complemented by illustrations consisting of high quality photographs and clear, unambiguous diagrams.
This book, the second volume in the Prosthodontics Section of the Quintessentials for Dental Practitioners series, is a valuable addition to the literature. Dentists who continue to provide complete dentures - but who feel that their skills in this challenging branch of dentistry need a timely boost - will find it particularly useful. Indeed, it may even provide the impetus to persuade a few more dentists to treat this very deserving group of patients.

D. Davis

\section{Oral and Maxillofacial Medicine}

\begin{tabular}{l}
\hline C. Scully \\
London: Wright, 2004 \\
price £38.99, pp556 \\
ISBN 0723610746 \\
\hline
\end{tabular}

Oral and Maxillofacial Medicine is a further contribution from one of the most prolific authors in the field of oral medicine, Professor Crispian Scully. The book, which is aimed at those interested in oral medicine and hospital dentistry, is organised in a very accessible format and comprises five colour coded sections. The text contains a vast amount of information and is commendably practical in its approach.

Section 1 addresses principles of patient management, embracing history taking, examination, investigation and treatment. This is a useful preface to the main text and is an area that is often overlooked.

Section 2 details the common signs and symptoms seen in the practice of oral medicine, including:

lymphadenopathy, dry mouth, halitosis, swellings, pain, red, white and pigmented lesions, neurological involvement and ulcers. Key points are identified for each topic. Here the reader is introduced to the first of many algorithms, which helpfully illustrate the text throughout. Inexperienced clinicians should take care to differentiate between the common and the rarer conditions shown in some of the more complex algorithms.

Section 3 addresses 'common and important oral conditions'. This, the largest of the five sections of the book, is organised alphabetically. The subsections follow the theme of an introduction, disease incidence, predisposing factors, aetiology, pathogenesis, clinical features, diagnosis and management. Additionally, useful sample patient information sheets are included. Some readers, however, may be surprised that denture-related stomatitis is described under a separate heading to oral candidiasis.

Section 4 deals with eponymous and 'other conditions', essentially a miscellany of relative rarities. Conditions are again arranged alphabetically, facilitating quick reference for uncommon entities. Section 5 constitutes relevant other systemic disorders and is devoted to HIV and iatrogenic disease.

Finally, two appendices tabulate information on oral manifestations of systemic disease and therapies for oral disease. As mentioned earlier, inexperienced clinicians should exercise caution with some of the less mainstream treatment regimes that are described.

Oral and Maxillofacial Medicine is a first class text. It is up to date and contains a wealth of easily accessible and practical information. It is well illustrated and the use of algorithms is particularly helpful. A variety of websites are included within the references

At a cost of less than $£ 40$, this book represents outstanding value. Senior dental students, hospital and general dental practitioners will all find it both an excellent source of reference and a thoroughly practical guide to oral medicine. 

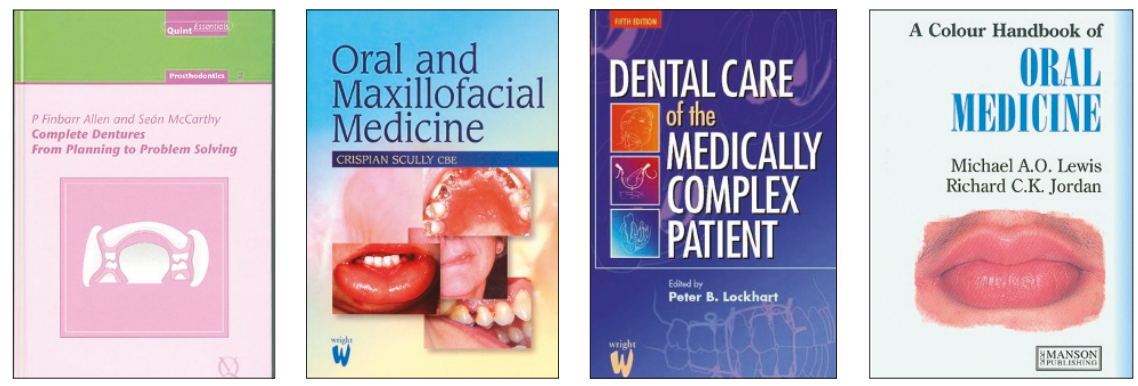

\section{Dental Care of the Medically Complex Patient}

\author{
J. Meechan, J. Nunn, Ed. P.B. Lockhart \\ London: Elsevier, 2003 \\ price $€ 23.99, \mathrm{pp} 472$ \\ ISBN 0723610908
}

This book is a handy-sized reference for dental practitioners who want easily accessible information regarding the relevance of medical conditions to the safe provision of dental treatment. It is written in an easily readable style which may have been enhanced still further by the introduction of some clinical photographs at pertinent points in the text.

The book contains nine chapters that are wide-ranging - from dental emergencies to maxillofacial prosthetics - and are followed by 11 appendices (some less than one page in length) with an equally wide-ranging remit.

Areas of controversy are dealt with authoritatively, eg. the debate for the need or otherwise for supplemental steroids for patients taking or who have taken steroids undergoing certain types of dental treatment. Although pointing out that adrenal crisis in the dental setting is extremely rare it is true that supplementation is often given because it is 'easy, inexpensive and nonthreatening to the patient in comparison with the potential outcome from an adrenal crisis'.

Certain areas differ from current UK practice in that the INR considered safe for dental extractions in patients taking warfarin is given as four. Indeed, this is recommended by some authorities, but current advice in the UK is 3.0 (Dental Practitioners' Formulary 2002-4).

Chapter 3 provides a good synopsis of oral medicine and ties in well with the more holistic medical feel of the book overall. The book usefully includes a series of examples of consultation requests and many of the examples will be familiar to readers.
My only criticism of this book is that it perhaps tries to cover too broad a canvas - there are sections detailing subjects such as maxillofacial trauma and topical fluoride concentrations. If the book had been limited strictly to the relevance of medical conditions on dental treatment it might have improved it.

Overall though, this is a very useful book and will be valuable to undergraduate and postgraduate students as well as practising clinicians in both hospital and general practice.

M. Greenwood

\section{A Colour Handbook of Oral Medicine}

M.A.O. Lewis, R.C.K. Jordan
London: Manson, 2004
price £29.95, pp176
ISBN 1840760338

This book aims to provide a clinician with a well illustrated text to aid the diagnosis and initial management of disorders relevant to oral medicine. I therefore assume the text is aimed at general dental practitioners.

An introductory chapter of the book provides information regarding the potential investigation of relevant disease. Seven further chapters each consider the disorders likely to give rise to a particular sign or symptom affecting the mouth.

The principal chapters all contain key points of diagnosis and list differential diagnoses of a given symptom or sign. Thereafter, the chapters discuss each disorder by providing short summaries of aetiology, pathogenesis, clinical features, diagnosis and management. Clinicians will find many good examples of common disorders such as squamous cell carcinoma, oral lichen planus, erythema multiforme and primary herpetic gingivostomatitis, together with less common disorders such as linear IgA disease, white sponge naevus and dyskeratosis congenita.

A considerable number of colour images of relevant lesions are provided throughout. Without doubt, the strength of the text lies in these images - which are generally of a high quality and demonstrate pertinent lesions well along with the succinct summaries provided of the clinical features of each disorder. Hence general dental practitioners are likely to gain useful and relevant clinical insight into the world of oral medicine. In addition, readers should be able to appreciate the spectrum of disease that may arise and its possible association with systemic disease.

There are some weaknesses in the text which may reflect the very rapid changes that occur in both medicine and oral medicine. For example, the treatment of oral dysplastic lesions should probably be more aggressive than stated, while the role of patch-testing in the diagnosis of lichenoid reaction remains questionable. Some of the other investigations and managements may be of limited benefit.

Not withstanding, this is a well written, well presented text that I am sure will be of interest, and clinical relevance, to general dental practitioners.

S.R. Porter 